\title{
Outcomes of bariatric surgery in men versus women: a matched observational cohort analysis
}

\section{Andrew C. Kennedy-Dalby, Basil J. Ammori \& Akheel A. Syed}

Faculty of Medical and Human Sciences, The University of Manchester and Salford Royal NHS Foundation Trust, Salford

\section{Introduction}

Obesity is a cause of significant mortality and morbidity in the UK with currently $26 \%$ of adults in the UK clinically obese ${ }^{1}$. Bariatric surgery has been shown to be an effective treatment for obesity, giving significant weight loss as well putting type 2 diabetes, hypertension, hyperlipidaemia and obstructive sleep apnoea (OSA) into remission. ${ }^{2,3,4}$ However there is a disparity in the number of men accessing bariatric services, with only $19 \%$ of bariatric patients being male. ${ }^{5}$ To aid our understanding of this disparity, it is important to ascertain whether metabolic outcomes after bariatric surgery differ between genders, as this may be a potential barrier for accessing treatment.

This study examined changes in several metabolic outcomes after bariatric surgery in adult males and females: Body Mass Index (BMI), Blood Pressure, Total Cholesterol: HDL ratio and HbA1c (in patients with a diagnosis of Type 2 Diabetes Mellitus). Further to these factors, this study also investigated changes in several clinical variables: the use of CPAP therapy for OSA, and the prescription of diabetic medications - with particular regard given to the use of Insulin before and after surgery. This will enable us to highlight any gender differences in the outcomes of bariatric surgery, which in turn will allow for a greater understanding of the disparity in access to bariatric surgical services in men and women.

\section{Materials and methods}

The study highlighted adult patients who underwent bariatric surgery at Salford Royal Hospital, Manchester in a three-year period. A number of variables were recorded: age at time of surgery, type of surgery, diabetes diagnosis and corresponding medications particularly insulin, $\mathrm{HbA1c}$, height, weight, systolic and diastolic blood pressure, total cholesterol: high-density lipoprotein (HDL) ratio, and OSA treated with CPAP. Data was recorded for 12 months, 6 months and 3 months prior to surgery as well as at the time of surgery to give a mean preoperative result. Outcomes following surgery were recorded at 6 weeks, 4 months, 12 months, 24 months and 36 months after surgery.

As there was a much greater number of women identified, data for all males was captured and matched to women of equal age ( \pm 5 years), BMI $\left( \pm 2 \mathrm{~kg} / \mathrm{m}^{2}\right)$, Type 2 Diabetes diagnosis, and consequent insulin therapy, and CPAP therapy for OSA.

Data was statistically analysed for significant difference between men and women by unpaired T-test. Fisher's tests were used to compare $2 \times 2$ tables of data for CPAP therapy and insulin use.

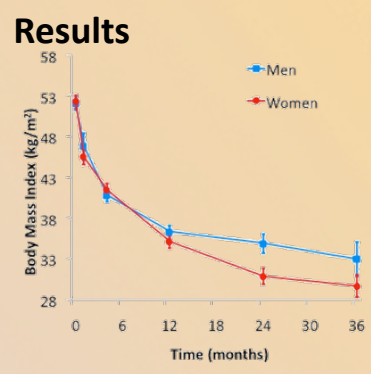

\begin{tabular}{lccc}
\hline & Men & Women & P \\
Age (years) & 46.4 & 46.1 & 0.850 \\
BMI $\left(\mathrm{kg} / \mathrm{m}^{2}\right)$ & $52.1( \pm 7.4)$ & $52.3( \pm 7.5)$ & 0.8858 \\
HbA1c $(\mathrm{mmol} / \mathrm{mol})$ & $71( \pm 19.8)$ & $61( \pm 19.8)$ & 0.0545 \\
Systolic BP $(\mathrm{mm} \mathrm{Hg})$ & $150( \pm 18.5)$ & $141( \pm 17.9)$ & 0.0399 \\
Diastolic BP $(\mathrm{mm} \mathrm{Hg})$ & $91( \pm 12.0)$ & $88( \pm 11.8)$ & 0.2444 \\
Total cholesterol: HDL & $4.5( \pm 1.4)$ & $4.1( \pm 1.2)$ & 0.1218 \\
\hline \multicolumn{4}{c}{ Table 1: Mean values for matched criteria in men and women }
\end{tabular}

80 men and 80 matched women were compared. There was no significant difference in BMI over the 36 month period, although women experienced a faster decrease in BMI compared to men and have statistically lower $B M I$ at 24 months $(P=0.0138)$, but not at 36 months $(P=0.2223)$. Whilst there is a significant difference in the change systolic blood pressure at 24 months $(P=0.0099)$, there is no corresponding significant difference in diastolic blood pressure $(P=0.7923)$, and both genders experienced a similar change in both systolic and diastolic blood pressure.
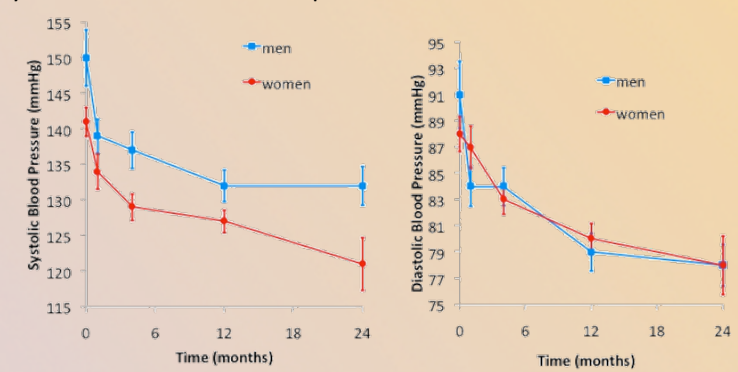

34 matched men and women had a diagnosis of type 2 diabetes prior to surgery. Of these, seven $(22.5 \%)$ were prescribed insulin. Following surgery, no men and one woman remained on insulin $(P=0.5256)$. In terms of glycaemic control, there is no statistical difference between men and women in HbAlc ( $P=0.1040$ at 24 months).

Similarly, there was no significant difference in the change in the total cholesterol :HDL ratio between men and women ( $P=0.8857$ at 24 months). $50 \%$ (40 of each gender) of patients in the study were using CPAP for OSA prior to surgery. Following surgery, only four women $(10 \%)$ and nine men (22.5\%) remained on CPAP $(P=0.2247)$.

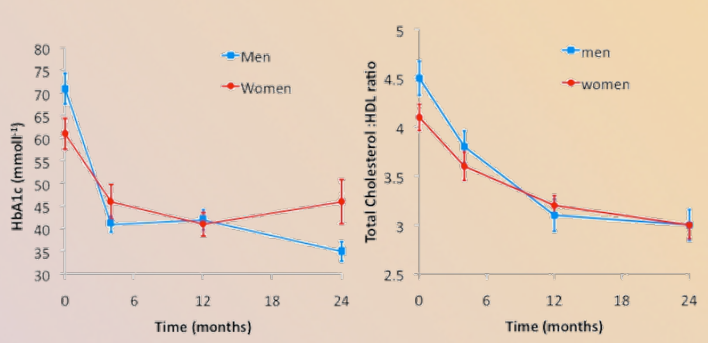

\section{Conclusions}

* Weight loss and metabolic outcomes after bariatric surgery are of similar magnitude in men compared to women.

* The use of bariatric surgery in eligible men should be encouraged.

* Further work is required to understand the current gender disparity in uptake of bariatric surgery.

\section{References}

1. Public Health England (2013). Trends in Obesity Prevalence. (http://noo.org.uk/NOO_about_obesity/trends). Accessed $27^{\text {th June }}$ 2013.
Sugerman H.J., Wolfe L.G., Sica D.A., Clore J.N. (2003). Diabetes and hypertension in severe obesity and effects of gastric-bypass induced weight loss. Annals of Surgery 237(6), 757-758. 3. Kardassis D., Grote L., Sjostrom L., Hedner J., Karason K. (2013). Sleep apnea modifies the long-term impact of surgically induced weight loss on cardiac function and inflammation. Obesity 21(4), 698-704. 4. Sjostrom C.D., Lissner L., Wedel H., Sjostrom L. (1999). Reduction in the incidence of diabetes, hypertension and lipid disturbances after intentional weight loss induced by bariatric surgery: the SOS Intervention Study. Obesity Research 7(5), 477-484. Buchwald H., Avidor Y., Braunwald E., Jensen M.D., Pories W., Fahrbach K., Schoelles K. (2004). Bariatric Surgery: A systematic review and meta-analysis. Journal of the American Medical Association 292(14), 1724-1737.

\section{Acknowledgments}

This study was undertaken at Salford Royal Hospital, Salford (July 2013). Permission was obtained from the Caldicott Guardian of the institution. 